\title{
Wandering the barren deserts of Iran: illuminating high mobility of the Asiatic cheetah with sparse data
}

\section{Mohammad S. Farhadinia}

Iranian Cheetah Society (ICS), POBox 14155-8549, Tehran, Iran

Wildlife Conservation Research Unit, Department of Zoology, University of Oxford, RecantiKaplan Centre, Tubney House, Oxfordshire, OX13 5QL, Oxford, U.K.

Corresponding author: msfarhadinia@wildlife.ir

\section{Navid Gholikhani}

Iranian Cheetah Society (ICS), POBox 14155-8549, Tehran, Iran (gho navid@yahoo.com)

\section{Pouyan Behnoud}

Iranian Cheetah Society (ICS), POBox 14155-8549, Tehran, Iran

(pouyaanbehnoud@yahoo.com)

\section{Kaveh Hobeali}

Iranian Cheetah Society (ICS), POBox 14155-8549, Tehran, Iran (kavehobeali@gmail.com)

\section{Atieh Taktehrani}

Iranian Cheetah Society (ICS), POBox 14155-8549, Tehran, Iran (a.taktehrani@gmail.com)

\section{Fatemeh Hosseini-Zavarei}


Iranian Cheetah Society (ICS), POBox 14155-8549, Tehran, Iran (fhosseini806@gmail.com)

\section{Morteza Eslami}

Iranian Cheetah Society (ICS), POBox 14155-8549, Tehran, Iran (morteza ed@yahoo.com)

Luke T. B. Hunter

Panthera, 8 West 40th Street, 18th Floor, New York 


\section{Abstract}

Mammalian species inhabiting arid landscapes exhibit various levels of mobility in order to cope with unpredictable spatiotemporal dispersion of resources. However, the space use and ranging behavior of many species in drylands is poorly understood, especially for large carnivores in Asia. Accommodating the long-distance movements of mammalian carnivores is a major challenge in conservation, particularly for threatened and highly mobile species. The Asiatic cheetah Acinonyx jubatus venaticus, a critically endangered subspecies found only in arid areas of Iran, exhibits high levels of mobility. In the absence of satellite collars, this paper outlines ranging patterns of the majority of the Asiatic cheetah's known population. A total of 17 independent cheetahs (11 males; 6 females) were detected with camera traps that were deployed on eight reserves between January 2011 and May 2016. The animals displayed extensive movements (up to $190 \mathrm{~km}$ between furthest known locations) and at least eight adult cheetahs were known to move between multiple reserves in central Iran. This mobility may follow a "nomadic" ranging pattern, a non-sedentary behavior with irregular timing and movement directions. The ranging behavior documented here must be considered when designing monitoring efforts to determine population and occupancy trends for this wide-ranging elusive carnivore. Although preliminary, our results show that improving protection beyond the current network of cheetah reserves, particularly along corridors, should be a top priority to safeguard the persistence of the Asiatic cheetah. 
Keywords: Acinonyx jubatus venaticus; nomadism; dryland; ranging pattern. 
Many mammals in dryland ecosystems show different types of long-range movement patterns (Harris et al., 2009). Various levels of mobility are associated with accessibility of unpredictable and spatially heterogeneous resources across landscapes (Durant et al., 2014). Long-distance movements of wildlife are important biological phenomena and their integration into conservation measures remains a major challenge (Kaczensky et al., 2011). Therefore, illuminating the underlying factors that shape various movement patterns is critical, particularly in case of endangered mobile species.

Central to efficient use of dryland resources, mobility is inevitable for desert dwelling wildlife (Durant et al., 2014). Presently, scientific knowledge about mammal migrations in Asia's arid ecosystems is limited largely to herbivores (Harris et al., 2009; Kaczensky et al., 2011). These species typically migrate long distances in the arid and semi-arid rangelands of central Asia to cope with the changing productivity and precipitation of their unpredictable environments (Olson et al., 2009; Singh et al., 2010). In contrast, our knowledge about the mobility of carnivores in Asian drylands is very limited. Grey wolves Canis lupus are reported to patrol large areas in Mongolian deserts (Kaczensky et al., 2008) and Asiatic cheetahs Acinonyx jubatus venaticus move exceptionally long distances in central Iran (Farhadinia et al., 2013). This may be due to the arid, resource-poor Iranian environments that require cheetahs to move long distances to fulfill their resource needs.

Ignoring extensive movements of highly mobile endangered species could result in misinterpretation of species' range changes. Researchers caution that total geographic range estimates (i.e. pooling observations over time) do not adequately reflect the more localized, highly concentrated occurrence of nomadic species at any particular point in time 
and may, therefore, exaggerate true occurrence (Teitelbaum et al., 2015). Thus, without adequate information on discrete spatial patterns, conservation efforts involving terrestrial migrations in fragile dryland ecosystems may easily be misguided and jeopardized.

With a population of fewer than 70 individuals left in Iran (Farhadinia, 2004), the Asiatic cheetah is critically endangered, prompting intensive conservation efforts in the country since 2001 (Hunter et al., 2007). However, their spatial ecology remains poorly understood. Home range sizes of cheetahs have shown large variation (see Houser et al., 2009 for more details), influenced by several factors, such as avoidance of other predators (Durant, 2000), following migratory prey species (Caro, 1994), low rainfall (Marker et al., 2008a) and human disturbance (Houser et al., 2009).

Asiatic cheetahs are known to roam large areas (Farhadinia et al., 2013) and occur at extremely low density (Hunter et al., 2007), both creating considerable challenges for research and monitoring. In the present paper we collated a photographic dataset of cheetahs detected in Iran to outline their spatial behavior. These data will add to our understanding from a previous case report (Farhadinia et al., 2013) with additional information collected across the majority of the cheetah's confirmed range in Iran. While our data are sparse, it is enhances our understanding of one of the rarest carnivore subspecies in the world. One aim was that our work may serve as a stimulus for future studies focusing on high mobility of endangered large carnivores in fragile drylands with highly heterogeneous resources.

Our study was conducted at 12 different cheetah reserves in the eastern half of Iran (Fig. 1 and Table 1). These landscapes are characterized by a mixture of arid plains, hilly terrain and undulating mountains. Vegetation predominantly comprises wormwood Artemisia sieberi 
and bean caper Zygophyllum sp. Persian ibex Capra aegagrus and wild sheep Ovis orientalis are the main wild ungulates whilst small numbers of chinkara Gazella bennettii and goitered gazelle Gazella subgutturosa also occur (Ziaie, 2008).

In order to monitor cheetahs, motion-triggered camera traps were deployed between January 2011 and May 2016. In total, 11 reserves with confirmed cheetah presence were selected to carry out camera trapping surveys. Eight reserves hosted systematic surveys, i.e. with an approximate average of 20 (ranging 10-43; Table 1) cameras that were strategically deployed for a period of three months, so at least one camera trap was present within two grids of $5 * 5 \mathrm{~km}$. The grid size corresponded to the smallest home ranges calculated for cheetahs in Africa (Houser et al., 2009). Additionally, during non-systematic survey periods, all investigated sites received fewer than five camera traps to place them at promising locations where cheetahs were known to visit regularly based on their tracks or previous survey efforts. These so-called opportunistic surveys were not continuous due to constraints in the number of camera traps available.

Multiple brands were deployed, including CamTrak (CamTrak South Inc., Watkinsville, Georgia, USA), PantheraCam (New York, NY 10018, USA) and Cuddeback Capture (Green Bay, WI, USA) models. During cold months, cameras were positioned along dry watercourses or sign posts, where cheetahs regularly visit for scent marking. In contrast, water sources were prioritized for setting camera traps during hot months. At one reserve, Dorouneh, we did not deploy camera traps, but photos taken by local rangers (always with a GPS point) enabled us to detect a single cheetah in multiple localities.

We compiled a photographic database containing all cheetah images taken by the camera traps or by photographers. We identified individuals by their unique spot patterns. For each 
cheetah, occurrence data were plotted using ArcMap software ver. 9.3 (ESRI, Redlands, CA, USA) and we calculated the $100 \%$ minimum convex polygon (MCP) encompassing all detections. We also calculated the maximum straight-line distance between any two successive detections for each individual.

Between 2011 and 2016, we identified a total of 22 cheetahs by means of photographs, most of which $(95.5 \% ; n=21)$ were detected through camera trap records. We excluded all dependent cubs and used 17 adults for our analysis (Table 2), including 11 males and 6 females. On average, males were detected over much longer time periods than females (36.4 \pm SE 4.4 versus $28.2 \pm$ SE 8.0 months; Table 2 ).

The mean MCP for all cheetahs measured 1656.2 \pm SE $556.1 \mathrm{~km}^{2}$ with males (Male: $1862.2 \pm$ $700.2 \mathrm{~km}^{2}$; Female: $1089.6 \pm$ SE $556.1 \mathrm{~km}^{2}$ ). This was likely an effect of camera trap placement at known scent marking locations which are visited more regularly by males ( Marker et al., 2008b; Marnewick et al., 2008). Almost one third of monitored cheetahs (i.e. seven individuals; $6 \mathrm{M}$ and $1 \mathrm{~F}$ ) were detected in multiple reserves (Table 2). Of these individuals, 5 (all males) were detected at sites $\geq 150 \mathrm{~km}$ apart, with a maximum distance of $190 \mathrm{~km}$, visiting between two and four different reserves (Fig.1 and Table 2).

Asiatic cheetahs exhibited high spatial mobility across the arid landscapes of eastern and central Iran. Roads through and/or between these landscapes apparently does not deter movement by cheetahs, although road accidents represent a major source of mortality for the species (Farhadinia et al., in press). Almost one third of all individuals showed interreserve movements, resulting in some of the longest distances ever recorded for the species (also see McVittie, 1979). In Ahaggar Cultural Park, Algeria, the mean maximum distance traveled by two male cheetahs based on camera-trapping data was nearly $45 \mathrm{~km}$ (Belbachir 
et al., 2015) while the farthest inter-observation distances of $>150 \mathrm{~km}$ were previously only published for male cheetahs living in another semi-arid desert landscape, the Namibian farmland (see McVittie, 1979). With the exception of Namibia's semi-arid farmlands where cheetah ranges can measure between 1344 to $2863 \mathrm{~km}^{2}$ (Marker et al., 2008b; Wachter et al., 2006), most spatial ecology studies in sub-Saharan Africa yielded comparatively smaller cheetah ranges (see Houser et al., 2009 for more details) than recorded here. In contrast, Belbachir et al. (2015) calculated a maximum home range of $1337 \mathrm{~km}^{2}$ based on $100 \% \mathrm{MCP}$ estimation of camera trap detections in the arid areas of the Sahara desert.

We acknowledge that home range estimation by means of camera trap records underestimates true range sizes significantly when compared with estimates derived from radio telemetry (Gil-Sanchez et al., 2011). Accordingly, our data should be regarded as a minimum estimate of the potential distances moved and range sizes occupied by Asiatic cheetahs. Cheetahs almost certainly ranged beyond our survey footprint, highlighting the need for expanding the scale of both monitoring and conservation efforts in Iran.

We do not yet understand enough about the factors that influence the cheetah's wide ranging behavior in Iran. Low habitat productivity is believed to be an important determinant because most of the cheetah's range in Iran receives $<150 \mathrm{~mm}$ annual precipitation (Modarres and da Silva, 2007), resulting in heterogeneous arid landscapes with considerable spatiotemporal variation in prey availability. Regardless of the cause, the present data provide little evidence for predictability in cheetah movement patterns, suggesting that Asiatic cheetahs may move nomadically. Nomadism is a non-sedentary behavior which does not necessarily entail a return journey and involves irregular timing and movement directions (Roshier and Reid, 2003). Unlike stable sedentary ranges and 
migration, nomadism lacks inter-year predictability and can vary among individuals among years (Mueller and Fagan, 2008). Asiatic cheetahs have slightly lower genetic variation than most African populations, thought to be the result of ancestral population divergence or a recent bottleneck (Charruau et al., 2011). Their current nomadic movements might act to limit the further loss heterogeneity by fostering high gene flow, as has been shown for Namibian cheetahs (Marker et al., 2008a). Consequently, maintaining the ability of individuals to travel large distances is essential if further genetic erosion of this tiny population is to be avoided. As for other cheetah subspecies occurring at very low density (Belbachir et al., 2015), understanding the Asiatic cheetah's spatial ecology will be essential to develop and implement effective monitoring strategies. For example, repeat sightings of the same individual (by different untrained observers) throughout its large resident home range can cause inflated local cheetah population estimates (Marker et al., 2008a).

Furthermore, conceptualization of geographic range size for a nomadic species could lead to the erroneous conclusion that it is safe from extinction (Teitelbaum et al., 2015). Accordingly, further exploring the different aspects of landscape use by cheetahs is crucial to improve management and conservation of the species in Iran's drylands.

Expanding upon earlier research (Farhadinia et al., 2013), this paper highlights the Asiatic cheetah's extremely high mobility. Our results emphasize the importance of continuous monitoring of this critically endangered predator at national scale in order to understand the spatial implications for its effective conservation as well as its status. Beyond discrete spatial patterns, the low number of Asiatic cheetahs that were detected constitutes a major concern, highlighting the importance of effective protection throughout the species' entire remaining range. 


\section{Acknowledgement}

We thank our collaborators, including the Iranian Department of the Environment (DoE), Conservation of Asiatic Cheetah Project (CACP) and our supportive funders, the Iran UNDP, Panthera, La Palmyre Zoo, Association Francaise des Parcs Zoologiques (AFdPZ), Prince Bernhard Nature Fund (PBNF), Stichting SPOTS, the People's Trust for Endangered Species (PTES), Parcs des Felins \& SOS and WWF INNO Program. Also, thank you to the surveyed reserves' game wardens for field assistance and data collection. 
Belbachir, F., Pettorelli, N., Wacher, T., Belbachir-Bazi, A., Durant, S.M., 2015. Monitoring rarity: the critically endangered Saharan cheetah as a flagship species for a threatened ecosystem. PloS one, 10(1), p.e0115136.

Caro, T.M., 1994. Cheetahs of the Serengeti Plains: group living in an asocial species. University of Chicago Press.

Charruau, P., Fernandes, C., Orozco-Terwengel, P., Peters, J., Hunter, L., Ziaie, H., Jourabchian, A., Jowkar, H., Schaller, G., Ostrowski, S., Vercammen, P., Grange, T., Schlötterer, C., Kotze, A., Geigl, E., Walzer, C., Burger, P., 2011. Phylogeography, genetic structure and population divergence time of cheetahs in Africa and Asia: evidence of long-term geographic isolates. Mol Ecol 20:706-724.

Durant, S.M., 2000. Living with the enemy: avoidance of hyenas and lions by cheetahs in the Serengeti. Behavioral Ecology, 11(6), pp.624-632.

Durant, S.M., Wacher, T., Bashir, S., Woodroffe, R., Ornellas, P., Ransom, C., Newby, J., Abáigar, T., Abdelgadir, M., El Alqamy, H., Baillie, J., 2014. Fiddling in biodiversity hotspots while deserts burn? Collapse of the Sahara's megafauna. Diversity and Distributions, 20(1), pp.114-122.

Farhadinia, M.S., 2004. The last stronghold: cheetah in Iran. Cat News, 40, pp.11-14.

Farhadinia, M.S., Akbari, H., Mousavi, S.J., Eslami, M., Azizi, M., Shokouhi, J., Gholikhani, N., HosseiniZavarei, F., 2013. Exceptionally long movements of the Asiatic cheetah Acinonyx jubatus venaticus across multiple arid reserves in central Iran. Oryx, 47(03), pp.427-430.

Farhadinia, M.S., Akbari, H., Eslami, M., Adibi, M.A., in press. The rarest cat in west Asia: a review of ecology and conservation status of Asiatic cheetah Acinonyx jubatus venaticus in Iran. Cat News Spcial Issue Iran Cats.

Gil-Sánchez, J.M., Moral, M., Bueno, J., Rodríguez-Siles, J., Lillo, S., Pérez, J., Martín, J.M., Valenzuela, G., Garrote, G., Torralba, B., Simón-Mata, M.Á., 2011. The use of camera trapping for estimating Iberian lynx (Lynx pardinus) home ranges. European Journal of Wildlife Research, 57(6), pp.1203-1211.

Harris, G., Thirgood, S., Hopcraft, J.G.C., Cromsigt, J.P.G.M., Berger, J., 2009. Global decline in aggregated migrations of large terrestrial mammals. Endangered Species Research, 7(1), pp.5576.

Houser, A.M., Somers, M.J., Boast, L.K., 2009. Home range use of free-ranging cheetah on farm and conservation land in Botswana. South African Journal of Wildlife Research, 39(1), pp.11-22.

Hunter, L., Jowkar, H., Ziaie, H., Schaller, G., Balme, G., Walzer, C., Ostrowski, S., Zahler, P., RobertCharrue, N., Kashiri, K., Christie, S., 2007. Conserving the Asiatic cheetah in Iran: launching the first radio-telemetry study. Cat News, 46, pp.8-11.

Kaczensky, P., Enkhsaikhan, N., Ganbaatar, O., Walzer, C., 2008. The Great Gobi B Strictly Protected Area in Mongolia-refuge or sink for wolves Canis lupus in the Gobi. Wildlife Biology, 14(4), pp.444-456.

Kaczensky, P., Kuehn, R., Lhagvasuren, B., Pietsch, S., Yang, W., Walzer, C., 2011. Connectivity of the Asiatic wild ass population in the Mongolian Gobi. Biological conservation, 144(2), pp.920-929. 
Marker, L.L., Dickman, A.J., Mills, M.G., Jeo, R.M., Macdonald, D.W., 2008a. Spatial ecology of cheetahs on north-central Namibian farmlands. Journal of Zoology, 274(3), pp.226-238.

Marker, L., Fabiano, E., Nghikembua, M., 2008b. The Use of Remote Camera Traps to Estimate Density of Freeranging Cheetahs in North-Central Namibia. Cat News, 49, pp.22-24.

Marnewick, K., Funston, P.J., Karanth, K.U., 2008. Evaluating camera trapping as a method for estimating cheetah abundance in ranching areas. South African Journal of Wildlife Research, 38(1), pp.59-65.

McVittie, R., 1979. Changes in the social behaviour of South West African cheetah. - Madoqua 2: 171-184.

Modarres R, da Silva, V.P.R., 2007. Rainfall trends in arid and semi-arid regions of Iran. Journal of Arid Environments 70: 344-355.

Mueller, T., Fagan, W.F., 2008. Search and navigation in dynamic environments-from individual behaviors to population distributions. Oikos, 117(5), pp.654-664.

Olson, K.A., Mueller, T., Bolortsetseg, S., Leimgruber, P., Fagan, W.F., Fuller, T.K., 2009. A mega-herd of more than 200,000 Mongolian gazelles Procapra gutturosa: a consequence of habitat quality. Oryx, 43(01), pp.149-153.

Roshier, D., Reid, J., 2003. On animal distributions in dynamic landscapes. Ecography, 26(4), pp.539544.

Singh, N. J., I. A. Grachev, A. B. Bekenov, Milner-Gulland, E. J., 2010. Tracking greenery in central Asia - the migration of the saiga antelope. Diversity and Distributions 16:663-675.

Teitelbaum, C.S., Fagan, W.F., Fleming, C.H., Dressler, G., Calabrese, J.M., Leimgruber, P., Mueller, T., 2015. How far to go? Determinants of migration distance in land mammals. Ecology letters, 18(6), pp.545-552.

Wachter, B., Schulze, S., Lonzer, J., Berger, A., Melzheimer, J., East, M., Selbert, S., Hofer, H., 2006. The use of data from VHF and GPS radio-collared cheetahs to decrease conflicts between cheetahs and farmers in Namibia. In Proceedings of the European Telemetry Conference (Vol. 26, pp. 556-567).

Ziaie, H., 2008. A field guide to the mammals of Iran 2nd Editio., Tehran: Iranian Center for Wildlife. 
Figure 1: Ranging patterns of identified cheetahs in central Iran. Each MCP code refers to the relevant cheetah ID in Table 2. Numbers in the map represent cheetah reserves in Iran: 1) Miandasht, 2) Khosh Yeilagh, 3) Touran, 4) Dorouneh, 5) Kavir, 6) Abbas Abad, 7) Siahkouh, 80 Dareh Anjir, 9) Kamki Bahabad, 10) Ariz, 11) Bafq, 12) Kalmand, 13) Naybandan, and 14) Darband-e-Ravar. 
Table 1: Details of the cheetah photographic surveys conducted in Iran between 2011 and 2016.

Systematic camera trapping survey

\begin{tabular}{l}
\hline Reserve Name \\
Miandasht \\
Dorouneh \\
Khosh Yeilaq \\
Bafq \\
Dareh Anjir \\
Ariz \\
Siahkouh \\
Abbas Abad \\
Kamki Bahabad \\
Naybandan \\
Darband-e-Ravar \\
Kavir
\end{tabular}

1
0
2
1
0
0
Opportunistic camera trapping survey

No. of camera stations

38 to 43

0

0

42

26

10

23

35

0

48

1

\section{No. of camera stations}

Surveyed area (MCP of camera traps in $\mathbf{~ k m}^{2}$ ) Systematic camera trapping survey 550 NA NA 600 325 350 300 940 0 450 760

0


Table 2: Movement data of individual cheetahs in Iran (2011-2016)

\begin{tabular}{|c|c|c|c|c|c|c|c|c|c|c|c|c|}
\hline No & $\begin{array}{l}\text { Cheetah } \\
\text { ID }\end{array}$ & Sex & $\begin{array}{l}\text { Site first } \\
\text { detected }\end{array}$ & $\begin{array}{l}\text { Maximum } \\
\text { inter- } \\
\text { reserve } \\
\text { movement } \\
(\mathbf{k m})\end{array}$ & $\begin{array}{l}\text { Maximum } \\
\text { intra- } \\
\text { reserve } \\
\text { movement } \\
\text { (km) }\end{array}$ & $\begin{array}{l}\text { Period of } \\
\text { movement }\end{array}$ & $\begin{array}{c}\mathrm{MCP} \\
\left(\mathrm{km}^{2}\right)\end{array}$ & $\begin{array}{l}\text { No. of } \\
\text { reserves } \\
\text { visited }\end{array}$ & $\begin{array}{l}\text { No. of stations } \\
\text { with positive } \\
\text { detection }\end{array}$ & $\begin{array}{c}\text { First } \\
\text { detection }\end{array}$ & $\begin{array}{c}\text { Last } \\
\text { detection }\end{array}$ & $\begin{array}{c}\text { No. of } \\
\text { detections }\end{array}$ \\
\hline 1 & M8 & $M$ & Dareh Anjir & NA & 41 & 10 & 91 & 1 & 3 & Nov-14 & Sep-15 & 17 \\
\hline 2 & M5 & $M$ & Dareh Anjir & 185 & 38 & 45 & 6700 & 4 & 19 & Dec-11 & Sep-15 & 58 \\
\hline 3 & M4 & $M$ & Dareh Anjir & 174 & 38 & 45 & 4900 & 3 & 21 & Dec-11 & Sep-15 & 57 \\
\hline 4 & M3 & $M$ & Dareh Anjir & 174 & 38 & 45 & 4900 & 3 & 20 & Dec-11 & Sep-15 & 57 \\
\hline 5 & $\mathrm{~F} 2$ & $F$ & Dareh Anjir & 150 & 47 & 33 & 3585 & 3 & 10 & Jun-09 & Mar-12 & 10 \\
\hline 6 & M6 & $M$ & Ariz & 74 & 12.5 & 3 & 806 & 3 & 9 & Dec-11 & Mar-12 & 19 \\
\hline 7 & F7 & $\mathrm{F}$ & Ariz & NA & 8.6 & 2 & NA & 1 & 2 & Jan-12 & Mar-12 & 3 \\
\hline 8 & M19 & M & Kavir & NA & 62 & 42 & 480 & 1 & 6 & Mar-11 & Sep-14 & 23 \\
\hline 9 & M18 & $\mathrm{M}$ & Miandasht & NA & 24.3 & 44 & 129.4 & 1 & 12 & Jul-12 & Mar-16 & 25 \\
\hline 10 & M16 & $M$ & Miandasht & 73 & 14.9 & 42 & 419 & 2 & 7 & Aug-12 & Feb-16 & 12 \\
\hline 11 & F14 & $F$ & Miandasht & 78 & 27.5 & 54 & 616.8 & 2 & 8 & Nov-11 & May-16 & 19 \\
\hline 12 & F15 & $\mathrm{F}$ & Miandasht & NA & 14.9 & 45 & 118.6 & 1 & 6 & Aug-12 & May-16 & 15 \\
\hline
\end{tabular}




\begin{tabular}{|c|c|c|c|c|c|c|c|c|c|c|c|c|}
\hline 13 & F17 & $\mathrm{F}$ & Miandasht & NA & 14.9 & 31 & 38 & 1 & 5 & Aug-12 & Mar-15 & 11 \\
\hline 14 & M12 & $M$ & Naybandan & NA & 14 & 45 & 31 & 1 & 5 & Dec-11 & Sep-15 & 4 \\
\hline 15 & M13 & $M$ & Naybandan & 190 & 20 & 45 & 1858 & 2 & 3 & Dec-11 & Sep-15 & 6 \\
\hline 16 & M9 & $M$ & Naybandan & NA & 25 & 34 & 170 & 1 & 17 & Nov-12 & Sep-15 & 26 \\
\hline 17 & NA & $\mathrm{F}$ & Dorouneh & NA & 8.6 & 3 & NA & 1 & 2 & Jun-12 & Sep-12 & 2 \\
\hline
\end{tabular}




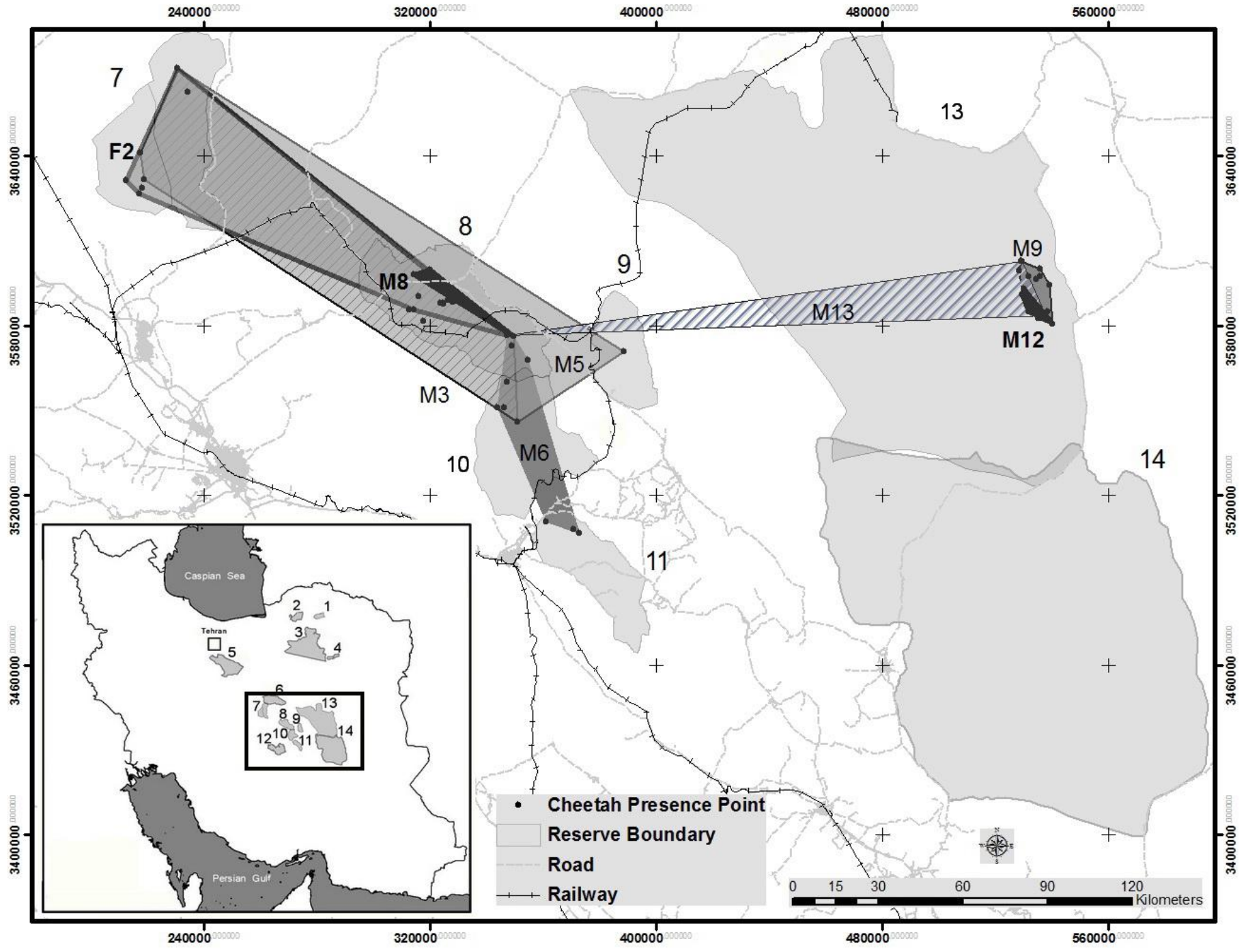

Fig 1 